\title{
Desempenho de novilhos superprecoces Angus, Devon e cruzas Angus x Devon $x$ Nelore em confinamento
}

\section{Performance of Angus, Devon and crossbred Angus x Devon x Nelore young steers in feedlot}

\author{
Carlos Santos Gottschall ${ }^{*}$; Leonardo Canali Canellas²; \\ Eduardo Tonet Ferreira ${ }^{3}$; Hélio Radke Bittencourt ${ }^{4}$
}

\section{Resumo}

\begin{abstract}
Foram analisados dados de 129 novilhos das raças Angus (AN, $n=45$ ), Devon (DV, $n=35$ ) e cruzas Angus x Devon x Nelore (CR, n=49), com peso médio inicial (PMI) de 277,60, 278,00 e 295,53 kg, respectivamente, confinados aos 11 meses de idade para abate aos 14 meses. As variáveis-resposta mensuradas foram: tempo médio de permanência (TMP); ganho de peso médio diário (GMD); ganho de peso total (GP); peso médio ao abate (PMA); peso médio de carcaça (PC); rendimento médio de carcaça (RC) e classificação de carcaça $(\mathrm{CC})$. As análises foram realizadas utilizando o PMI como co-variável, visto que este foi maior $(\mathrm{p}<0,01)$ para os $\mathrm{CR}$ em relação aos AN e DV. As variáveis GMD, TMP e CC não apresentaram diferenças significativas entre os grupos analisados $(\mathrm{p}>0,05)$. Animais $C R$ apresentaram maior $(\mathrm{p}<0,05)$ PMA $(375,04$ $\mathrm{kg})$ do que animais DV $(359,40 \mathrm{~kg})$, enquanto $\mathrm{AN}(365,31 \mathrm{~kg})$ não diferiram $(\mathrm{p}>0,05)$ de DV e CR. O PC $(\mathrm{p}<0,05)$ e o $\mathrm{RC}(\mathrm{p}<0,01)$ foram maiores para $\mathrm{CR}(191,12 \mathrm{~kg}$ e $50,99 \%)$ em relação à $\mathrm{AN}(179,81 \mathrm{~kg}$ e 49,73\%) e DV (177,23 kg e 49,41\%), respectivamente, que não apresentaram variações significativas entre si. Novilhos Angus e Devon obtiveram resultados semelhantes em todas as variáveis analisadas. Animais cruza Angus x Devon x Nelore apresentaram maior peso e rendimento de carcaça do que animais puros. Palavras-chave: Novilhos britânicos, Angus, Devon, confinamento, terminação
\end{abstract}

\begin{abstract}
The study was based on data from 129 beef steers from three genetic groups: Angus (AN, n=45), Devon $(\mathrm{DV}, \mathrm{n}=35)$ and Crossbred Angus $\mathrm{x}$ Devon $\mathrm{x}$ Nelore $(\mathrm{CB}, \mathrm{n}=49)$, with an average beginning weight $(\mathrm{ABW})$ of $277.60,278.00$ and $295.53 \mathrm{~kg}$, respectively. Those animals were in a feedlot with 11 months of age, to be slaughtered at 14 months. The parameters analyzed were: days on feed (DF), average weight gain (AWG), total weight gain (TWG), average slaughter weight (SW), average carcass weight (CW), average carcass yield (CY) and carcass classification (CC). The analysis were done using the ABW as a covariate because of these variable in the group $\mathrm{CB}$ were higher $(\mathrm{p}<0,01)$ than $\mathrm{AN}$ and $\mathrm{DV}$ groups. The variables $\mathrm{AWG}, \mathrm{DF}$ and $\mathrm{CC}$ did not show difference among the groups ( $>>0.05)$. CB animals got a higher $\mathrm{SW}(375.04 \mathrm{~kg})$ than DV (359.40) $(\mathrm{p}<0.05)$, while $\mathrm{AN}(356.31 \mathrm{~kg})$ did not differ from $\mathrm{DV}$ and $\mathrm{CB}(\mathrm{p}>0.05)$. The $\mathrm{CW}(\mathrm{p}<0.05)$ and $\mathrm{CY}(\mathrm{p}<0.01)$ were higher to CB (191.12 kg and 50.99\%) than AN (179.81 kg and 49.73\%) and DV (177.23 kg and 49.41\%), respectively. Angus and Devon beef steers had similar results in all variables analyzed. Crossbred animals presented greater carcass weight and carcass yield than the others groups.
\end{abstract}

Key words: British beef steers, Angus, Devon, feedlot, finishing cattle.

\footnotetext{
1 Médico Veterinário, MSc. Professor adjunto da Faculdade de Medicina Veterinária, ULBRA - Canoas/RS. E-mail: carlosgott@cpovo.net

2 Acadêmico do curso de Medicina Veterinária, ULBRA/RS. Bolsista de Iniciação Científica, BIC/FAPERGS.

3 Acadêmico do curso de Medicina Veterinária, ULBRA/RS. Bolsista de Iniciação Científica, PROICT/ULBRA

4 Estatístico, MSc. Faculdade de Matemática, PUCRS

* Autor para correspondência
} 


\section{Introdução}

A pecuária de corte no Brasil caracteriza-se pelos baixos índices de produção e pela baixa rentabilidade econômica. Ao se buscarem melhores índices de produtividade, surge a necessidade de redução da idade de abate dos animais (VAZ; RESTLE; PACHECO, 2002). Conforme Neumann, et al. (2002) o abate de novilhos aos 14 meses pode ser uma alternativa para aumentar o giro de capital e para modificar o sistema de organização da propriedade, pois a partir do momento em que esses terneiros deixam de ocupar espaço no campo, outras categorias animais são beneficiadas com maiores áreas de pastagem.

Myers et al. (1999) afirmam que existe uma crescente exigência do consumidor por carne de alta qualidade, e que, portanto, o produtor deve ser capaz de produzi-la com eficiência. De acordo com Restle e Vaz (1999), ao reduzir a idade de abate, o consumidor é beneficiado com uma carne de melhor qualidade e o produtor com um aumento na eficiência do seu sistema de produção.

De uma maneira geral, o peso de carcaça exigido pelos frigoríficos fica em torno dos $230 \mathrm{~kg}$. Entretanto, carcaças com menor peso estão sendo gradativamente aceitas no mercado interno, pois os consumidores estão associando pesos mais leves de carcaça como sendo de animais mais jovens e, portanto, carne de melhor qualidade (COSTA et al., 2002). No entanto, para que seja alcançado um grau de deposição de gordura adequado em carcaças leves, torna-se necessária a utilização de raças precoces. Segundo MOLETTA; RESTLE (1996), as raças britânicas são conhecidas pela rápida deposição de gordura e pelo bom desenvolvimento muscular, refletindo em uma conformação de carcaça adequada. Nesse sentido, muitos trabalhos de pesquisa têm sido conduzidos, objetivando avaliar o desempenho tanto de animais puros quanto de animais cruzados (FLORES, 1997; HUFFMANN et al., 1990; JOHNSTON; THOMPSON; HAMMOND, 1992; RESTLE; VAZ; QUADROS, 1999; SHERBECK et al., 1995).
O cruzamento é uma prática que permite o aproveitamento da capacidade genética dos animais, possibilitando o uso da complementaridade de raças (PADUA et al., 2004). Conforme Restle e Vaz (1999), no estudo entre raças e cruzamentos, é denominado como heterose o desvio que animais cruzados apresentam, para determinada característica, em relação à média das raças que originaram o cruzamento, em condições idênticas de meio. Com isso, o cruzamento explora a heterose que se manifesta devido aos efeitos de dominância, tais como menor conversão alimentar, maior taxa de ganho de peso e melhor rendimento e qualidade de caraça, entre e dentro das raças (PEROTTO et al., 2000). O objetivo do trabalho foi analisar o desempenho em confinamento e o rendimento de carcaça de novilhos Angus, Devon e cruzas Angus x Devon x Nelore abatidos aos 14 meses de idade.

\section{Material e Métodos}

O trabalho foi realizado entre 13/9 e 31/12/2005 em uma propriedade particular localizada no município de Cristal/RS. Foram analisados dados de 129 novilhos britânicos e cruzas [base britânica (Angus e Devon) com diferentes graus de sangue zebuíno (Nelore)], confinados aos 11 meses de idade, para abate aos 14 meses. Foram formados três lotes: AN (novilhos Angus puros, $\mathrm{n}=45$ ); DV (novilhos Devon puros, $n=35$ ); e CR (novilhos cruzas, $n=49$ ). Os animais eram provenientes do rebanho comercial da propriedade, e anteriormente ao confinamento receberam o mesmo tipo de manejo. O peso médio inicial (PMI) foi de 277,60, 278,00 e 295,53 kg, respectivamente para $\mathrm{AN}, \mathrm{DV}$ e $\mathrm{CR}$.

Os animais dos diferentes lotes foram confinados em piquetes distintos, a céu aberto, recebendo alimentação em cochos com espaço linear mínimo de $50 \mathrm{~cm}$ por novilho. A dieta, fornecida duas vezes ao dia, foi ajustada conforme National Research Council (1996) para GMD de 1,0 kg/dia. A composição da dieta variou ao longo do período de confinamento, sendo ajustada mensalmente conforme 
a variação de peso dos animais. Os ingredientes utilizados em proporções variáveis foram silagem de sorgo, quirela de arroz, resíduos de peneiras da prélimpeza de arroz, grão de sorgo, farelo de soja e uréia. A dieta continha níveis médios de $13 \%$ de proteína bruta e $70 \%$ de nutrientes digestíveis totais (NDT) nos primeiros 30 dias de confinamento, e 12\% de proteína bruta e $70 \%$ de NDT a partir do $2^{\circ}$ mês. A relação volumoso : concentrado também variou, sendo após os primeiros 12 dias de adaptação de 60 : 40. Após 45 dias a relação era de 40 : 60, com a utilização de maior quantidade de resíduos de menor qualidade e custo, mas sempre mantendo os níveis mínimos exigidos pelo National Research Council (1996). A ingestão de matéria seca foi estimada em $3 \%$ do peso vivo dos animais e assim oferecida. Os animais tiveram acesso ad libidum durante todo o período a uma mistura mineral contendo $80 \mathrm{~g}$ de fósforo por $\mathrm{kg}$.

Os novilhos foram pesados por ocasião da entrada no confinamento, posteriormente a cada 30 dias em média para avaliação do desenvolvimento e para ajustes à dieta, e no momento das vendas. As pesagens ocorreram pela manhã, anteriormente à colocação do alimento no cocho. Os abates ocorriam de forma escalonada, a medida que os animais atingiam o escore de condição corporal 7 na escala de 1 a 9 , representa $26,4 \%$ de gordura corporal na composição da carcaça (NATIONAL RESEARCH COUNCIL, 1996). Não havia um peso mínimo de carcaça pré-estabelecido.

As variáveis-resposta utilizadas foram Tempo Médio de Permanência (TMP), Ganho de Peso Médio Diário (GMD), Ganho de Peso Total (GP), Peso Médio ao Abate (PMA), Peso Médio de Carcaça (PC), Rendimento Médio de Carcaça (RC) e Classificação de Carcaça (CC). O PC foi obtido através das pesagens das carcaças frias. O RC foi calculado a partir de pesagens realizadas na propriedade, anteriormente ao carregamento dos animais. A CC é um método subjetivo utilizado no frigorífico para a avaliação da qualidade das carcaças, sendo M (média), B (boa) e O (ótima). Este método, subjetivo, desenvolvido pelo frigorífico, leva em consideração aspectos qualitativos como conformação, maturidade, lesões, coloração e principalmente uniformidade da cobertura de gordura. Para CC foram utilizados dados de 120 animais, pois em um abate de 9 animais o avaliador não pode estar presente. Para as demais características foram incluídos os dados dos 129 novilhos.

A análise estatística foi realizada no programa SPSS, consistindo da utilização do teste Qui-quadrado para comparação da CC entre as raças. Para as demais variáveis foi utilizado o teste ANOVA, sendo a diferença entre médias testada pelo teste de Tukey para significância de 1 e 5\%. As análises foram realizadas utilizando o PMI como co-variável, visto que este foi maior $(\mathrm{p}<0,01)$ para os animais $\mathrm{CR}$ em relação aos AN e DV. Com isso procurou-se desconsiderar o efeito do PMI sobre as outras variáveis.

\section{Resultados e Discussão}

$\mathrm{Na}$ Tabela 1 podem ser visualizados dados referentes a PMI, GMD, TMP, GP, e PMA para os diferentes grupos analisados. O PMI foi maior $(\mathrm{p}<0,01)$ para $\mathrm{CR}$ em relação à $\mathrm{AN}$ e $\mathrm{DV}$, que não diferiram entre si, indicando que novilhos cruzas tendem a ser mais pesados em relação a novilhos puros da mesma idade. Trabalhando com novilhos britânicos e cruzados de mesma idade terminados em confinamento, Restle, Quadros e Vaz (2000), verificaram resultados semelhantes. Os autores relataram um maior peso inicial para novilhos cruzas Hereford x Nelore em relação aos Hereford puros, atribuindo esse resultado a heterose entre os grupos raciais, a exemplo do que ocorreu no presente trabalho. 
Tabela 1. Peso Médio Inicial (PMI), Ganho de Peso Médio Diário (GMD), Tempo Médio de Permanência (TMP), Ganho de Peso Total (GP) e Peso Médio ao Abate (PMA), dos grupos Angus (AN), Devon (DV) e Cruzas (CR), seguidos das respectivas médias.

\begin{tabular}{lcccc}
\hline & AN & DV & CR & Total/Média \\
\hline N & 45 & 35 & 49 & 129 \\
PMI (kg) & $277,60^{\mathrm{a}}$ & $278,00^{\mathrm{a}}$ & $295,53^{\mathrm{b}}$ & 284,52 \\
GMD (kg/dia) & 0,98 & 0,94 & 0,93 & 0,95 \\
TMP (dias) & 90,02 & 87,40 & 85,29 & 87,51 \\
GP (kg) & $87,71^{\mathrm{a}}$ & $81,40^{\mathrm{ab}}$ & $79,51^{\mathrm{b}}$ & 82,89 \\
PMA (kg) & $365,31^{\mathrm{ab}}$ & $359,40^{\mathrm{a}}$ & $375,04^{\mathrm{b}}$ & 367,40 \\
\hline
\end{tabular}

a,b na linha, diferem significativamente $(\mathrm{p}<0,05)$.

O GMD não apresentou diferenças estatísticas significativas $(\mathrm{p}>0,05)$ entre os grupos analisados. Resultados semelhantes são reportados por Restle, Quadros e Vaz (2000), que não verificaram diferenças estatísticas quanto ao GMD de novilhos puros da raça Hereford, quando comparados a novilhos com $38 \%$ e $50 \%$ de sangue Nelore, todos confinados por 126 dias. Huffmann et al. (1990), trabalhando com novilhos de diferentes genótipos Angus e Brahman, verificaram GMD semelhantes para novilhos Angus puros (1,58 kg/dia) e 3/4 Angus x Brahman (1,65 kg/dia). Os autores relatam, no entanto, que quando a proporção aumentou para $1 / 2 \mathrm{e}$ $3 / 4$ de sangue zebuíno, o GMD foi maior para os cruzas $(1,76$ e $1,77 \mathrm{~kg} / \mathrm{dia}$, respectivamente) em relação aos puros. Confinando novilhos superprecoces de diferentes grupos genéticos, Souza (2006), relata um maior GMD para animais $1 / 2$ sangue Angus x Nelore $(1,57 \mathrm{~kg} / \mathrm{dia})$ em relação aos Angus puros $(1,31 \mathrm{~kg} /$ dia), atribuindo esses resultados à complementaridade ou heterose das raças Angus e Nelore.

O TMP também foi semelhante $(\mathrm{p}>0,05)$ para os três grupos analisados. Huffmann et al. (1990) obtiveram maior TMP para os Angus puros em relação aos cruzados, possivelmente devido ao menor GMD obtido pelos novilhos puros naquele experimento. Costa et al. (2002), trabalhando com novilhos Angus com PMI de $189 \mathrm{~kg}$, relatam um TMP de 144 dias em confinamento para abate aos 13 meses de idade. Nesse sentido, Gottschall (1999) afirma que quando manejados corretamente e alimentados para seu ponto ideal de terminação, novilhos de diferentes grupos genéticos terão desempenhos similares em períodos de tempo distintos.

O GP em confinamento foi maior $(\mathrm{p}<0,05)$ para o grupo AN em relação ao grupo CR. No entanto, DV não diferiu de $\mathrm{AN}$ e CR nessa variável. Apesar dos grupos analisados não terem diferido estatisticamente quanto ao GMD e ao TMP, numericamente os novilhos AN obtiveram maiores valores para essas variáveis em relação aos CR. Portanto, o fato do GP ser resultado da multiplicação do ganho de peso diário pelo número de dias em confinamento, fez com que o grupo AN apresentasse maior GP em relação ao grupo CR.

O grupo $\mathrm{CR}$ obteve maior $(\mathrm{p}<0,05)$ PMA em relação ao $\mathrm{DV}$, enquanto $\mathrm{AN}$ não diferiu de $\mathrm{DV}$ e CR neste parâmetro, indicando que o maior PMI dos CR gerou um maior PMA em relação aos DV, visto que o GP dos dois grupos foi similar. Do mesmo modo, Restle, Quadros e Vaz (2000), relatam maior PMA para novilhos $1 / 2$ Hereford $x$ Nelore em relação aos Hereford puros. Esses resultados podem ser 
atribuídos ao efeito da heterose, que determina um maior peso para animais com sangue zebuíno em relação a animais britânicos puros da mesma idade. Os novilhos AN, por sua vez, não diferiram $(\mathrm{p}>0,05)$ dos DV, pois o PMI e o GP foram semelhantes para as duas raças, indicando que novilhos Angus e Devon podem alcançar o grau de terminação adequado e serem abatidos com pesos similares. Costa et al. (2002) relatam resultados similares, com PMA de $370,0 \mathrm{~kg}$ para novilhos Angus abatidos aos 14 meses de idade. Trabalhando com novilhos Devon do pósdesmama até o abate, Johnston, Thompson e Hammond (1992) obtiveram PMA de 376,6 kg.

Os resultados obtidos para PMA - semelhantes para $\mathrm{AN}$ e $\mathrm{CR}$ - podem ser atribuídos ao maior GP apresentado por AN em relação a CR. Ou seja, apesar do PMI ter sido maior para os cruzas, o maior GP permitiu aos Angus um PMA semelhante aos cruzas. Resultados superiores para PMA são relatados por Souza (2006), que obteve para novilhos Angus puros e $1 / 2$ Angus $x$ Nelore abatidos aos 14 meses, PMA de 472,20 kg e 506,40 kg, respectivamente. $\mathrm{O}$ autor verificou diferença estatística entre esses dois grupos, sendo o maior peso ao abate dos novilhos cruzas, resultante do efeito genético aditivo entre as raças Angus e Nelore, como já foi mencionado anteriormente.

$\mathrm{O}$ peso e o rendimento de carcaça são características importantes, pois estão associados diretamente com o valor comercial do animal (COSTA et al., 2002), e podem ser visualizados na Tabela 2. O PC e o RC foram maiores $(\mathrm{p}<0,05 \mathrm{e}$ $\mathrm{p}<0,01$, respectivamente) para $\mathrm{CR}$ em relação à $\mathrm{AN}$ e DV, que não apresentaram variações significativas entre si $(p>0,05)$ quanto a esses parâmetros.

Tabela 2. Peso Médio de Carcaça (PC) e Rendimento Médio de Carcaça (RC), dos grupos Angus (AN), Devon (DV) e Cruzas (CR), seguidos das respectivas médias.

\begin{tabular}{lcccc}
\hline & AN & DV & CR & Média \\
\hline $\mathrm{N}$ & 39 & 49 & 32 & 120 \\
$\mathrm{PC}(\mathrm{kg})$ & $179,81^{\mathrm{a}}$ & $177,23^{\mathrm{a}}$ & $191,12^{\mathrm{b}}$ & 183,74 \\
$\mathrm{RC}(\%)$ & $49,73^{\mathrm{A}}$ & $49,41^{\mathrm{A}}$ & $50,99^{\mathrm{B}}$ & 50,2 \\
\hline
\end{tabular}

$a, b$ na linha, diferem significativamente $(p<0,05)$.

A,B na linha, diferem significativamente $(\mathrm{p}<0,01)$.

Costa et al. (2002) verificaram, para novilhos Angus abatidos aos 14 meses, PC e RC de 181,56 $\mathrm{kg}$ e $53,4 \%$ respectivamente, sendo o resultado obtido para RC superior aos atingidos para os três grupos no presente trabalho. Resultados semelhantes foram relatados por Menezes et al. (2005), que verificaram um rendimento de carcaça 4,98\% superior para animais cruzados (europeu x zebuíno) em relação a animais puros europeus. Trabalhando com novilhos Hereford x Nelore Restle, Vaz e Quadros (1999) também verificaram maior rendimento de carcaça à medida que aumentava a proporção de genes Nelore nos genótipos produzidos pelo cruzamento com a raça Hereford. Huffmann et al. (1990) não verificaram diferenças significativas quanto ao rendimento de carcaça de novilhos Angus comparados a novilhos $3 / 4$ e $1 / 2$ Angus x Nelore.

A Tabela 3 mostra a classificação das carcaças dos animais dos diferentes grupos analisados. Não houve diferença estatística significativa $(p>0,05)$ quanto à $\mathrm{CC}$, indicando que a raça ou o cruzamento não exerceu influência sobre a qualidade das carcaças. Observa-se que a maioria das carcaças foi classificada como "boa" ou "ótima". Portanto, novilhos superprecoces de diferentes raças ou cruzas podem apresentar carcaças semelhantes e bem acabadas, desde que sejam alimentados conforme suas necessidades nutricionais e abatidos no ponto ideal de terminação. 
Tabela 3. Classificação das carcaças, Média (M), Boa (B) e Ótima (O) de animais Angus, Devon e Cruzas, submetidos ao confinamento.

\begin{tabular}{|c|c|c|c|c|c|c|c|}
\hline & & $\mathrm{N}$ & $(\%)$ & & & & \\
\hline & $\mathrm{M}$ & & B & & $\mathrm{O}$ & & otal \\
\hline $\mathrm{AN}$ & $\begin{array}{ll}0 & (0,0)\end{array}$ & 29 & $(74,4)$ & 10 & $(25,6)$ & 39 & $(100)$ \\
\hline DV & $4 \quad(12,5)$ & 16 & $(50,0)$ & 12 & $(37,5)$ & 32 & (100) \\
\hline CR & $3 \quad(6,1)$ & 34 & $(69,4)$ & 12 & $(24,5)$ & 49 & (100) \\
\hline Total & $7 \quad(5,8)$ & 79 & $(65,8)$ & 34 & $(28,3)$ & 120 & (100) \\
\hline
\end{tabular}

Não houve diferença estatística significativa $(\mathrm{p}>0,05)$.

\section{Conclusão}

Novilhos Angus e Devon obtiveram resultados semelhantes em todas as variáveis analisadas. Animais cruzas apresentaram maior peso e rendimento de carcaça do que animais puros. Novilhos de diferentes raças ou cruzas, quando alimentados conforme suas necessidades e abatidos no seu ponto ideal de terminação, apresentam desempenhos semelhantes e produzem carcaças de boa qualidade.

\section{Referências}

COSTA, E. C.; RESTLE, J.; PASCOAL, L. L.; VAZ, F. N.; ALVES FILHO, D. C.; ARBOITTE, M. Z. Desempenho de novilhos Red Angus superprecoces, confinados e abatidos com diferentes pesos. Revista Brasileira de Zootecnia, Viçosa, v.31, n.1, 2002.

FLORES, J. L. C. Desempenho em confinamento e características de carcaça e da carne de bovinos de diferentes grupos genéticos abatidos aos quatorze meses. 1997. Dissertação (Mestrado em Zootecnia) Universidade Federal de Santa Maria, Santa Maria.

GOTTSCHALL, C. S. Suplementação animal em pastagens: manejo, produtividade e custos de produção. In: CICLO DE PALESTRAS EM PRODUÇÃO E MANEJO DE BOVINOS DE CORTE: MANEJO E UTILIZAÇÃO SUSTENTÁVEL DE PASTAGENS, 4., 1999, Canoas. Anais... Canoas: Ed. ULBRA, 1999. p.57-90.

HUFFMANN, R. D., Williams, S. E., HARGROVG, D. D., JOHNSON, D. D.; MARSHALL, T. T. Effects of percentage Brahman and Angus breeding, age-season of feeding and slaughter end point on feedlot performance and carcass characteristics. Journal of Animal Science, Champaign, v.68, p.2243-2252, 1990.

JOHNSTON, J. D., THOMPSON, M. J.; HAMMOND, K. Additive and nonadditive differences in postweaning growth and carcass characteristics of devon, hereford, and reciprocal-cross steers. Journal of Animal Science, Champaign, v.70, p.2688-2694, 1992.

MENEZES, L. F. G.; RESTLE, J.; BRONDANI, I. L.; BRONDANI, I. L.; ALVES FILHO, D. C.; KUSS, F.; SILVEIRA, M. F.; AMARAL, G. A. Carcass characteristics of feedlot finished steers from advanced generations of rotational crossbreeding between Charolais and Nellore breeds. Revista Brasileira de Zootecnia, Viçosa, v.34, n.3, p.934-945, 2005.

MOLETTA, J. L.; RESTLE, J. Características de carcaça de novilhos de diferentes grupos genéticos terminados em confinamento. Revista Brasileira de Zootecnia, Viçosa, v.25, n.5, p.876-888, 1996.

MYERS, S. E., FAULKNER, D. B., IRELAND, F. A., BERGER, L. L.; PARRETT, D. F. Production systems comparing early weaning to normal weaning with or without creep feeding for beef steers. Joural of Animal Science, Champaign, v.77, p.300-310, 1999.

NATIONAL RESEARCH COUNCIL. (NRC). Nutrient requeriments of beff cattle. 7.ed. Washington: National Academy Press, 1996.

NEUMANN, M; RESTLE, J.; ALVES FILHO, D. C.; BRONDANI, I. L.; MENEZES, L. F. G. Resposta econômica da terminação de novilhos em confinamento, alimentados com silagens de diferentes híbridos de sorgo (sorghum bicolor, L.Moench). Ciência Rural, Santa Maria, v.32, n.5, p.849-854, 2002. 
PADUA, J. T.; MAGNABOSCO, C. U.; SAINZ, R. D.; MIYAGI, E. S.; PRADO, C. S.; RESTLE, J.; RESENDE, L. S. Genótipo e condição sexual no desempenho e nas características de carcaça de bovinos de corte superjovens. Revista Brasileira de Zootecnia, Viçosa, v.33, n.6, p.2330-2342, 2004. Suplemento 3.

PEROTTO, D., MOLETTA, J. L., OLIVEIRA, J. E. P.; LESSKIU, C. Consumo e conversão alimentar de machos bovinos inteiros Charolês, Caracu e cruzamentos recíprocos em confinamento. Revista Brasileira de Zootecnia, Viçosa, v.29, n.1, p.108-116, 2000.

RESTLE, J., QUADROS, A. R.; VAZ, F. N. Terminação em confinamento de novilhos de diferentes genótipos de Hereford x Nelore. Revista Brasileira de Zootecnia, Viçosa, v.29, n.1, p.125-130, 2000.

RESTLE, J., VAZ, F. N. Confinamento de bovinos definidos e cruzados. In: LOBATO, J. F. P.; BARCELLOS, J. O. J.; KESSLER, A. M. Produção de bovinos de corte. Porto Alegre: ED. IPPUCRS, 1999. p.141-168.
RESTLE, J.; VAZ, F. N.; QUADROS, A. R. B. Características de carcaça e da carne de novilhos de diferentes genótipos de Hereford x Nelore. Revista Brasileira de Zootecnia, Viçosa, v.28, n.6, p.1245-1251, 1999.

SHERBECK, J. A.; TATUM, T. G.; FIELD, J.B.; MORGAN, J. B.; SMITH, G. C. Feedlot performance, carcass traits, and palatability traits of Hereford and Hereford Brahman steers. Journal of Animal Science, Champaign, n.73, v.12, p.3613-3620, 1995.

SOUZA, A. A., Efeito de grupo genético sobre desempenho e características de carcaça de bovinos confinados com altos níveis de concentrado. Disponível em: $<$ http://www.beefpoint.com.br $>$. Acesso em: 07 maio 2006.

VAZ, F.N., RESTLE, J., PACHECO, P.S. et al. Características de carcaça e da carne de novilhos superprecoces de três grupos genéticos, gerados por fêmeas de dois anos. Revista Brasileira de Zootecnia, Viçosa, v.31, n.5, p.19731982, 2002. 